Pacific Journal of Mathematics

A NOTE ON THE FUNDAMENTAL GROUP OF A COMPACT 


\title{
A NOTE ON THE FUNDAMENTAL GROUP OF A COMPACT MINIMAL HYPERSURFACE
}

\author{
Gregory J. GaLloway
}

\begin{abstract}
In this paper we generalize a well-known result of Frankel which relates the fundamental group of a complete Riemannian manifold with positive Ricci curvature to the fundamental group of a compact immersed minimal hypersurface. Here we consider the situation in which the Ricci curvature of the ambient manifold is only assumed to be nonnegative, and show that the conclusion of Frankel's theorem can fail only under special circumstances.
\end{abstract}

1. Introduction. The theory of minimal surfaces has provided a powerful tool for studying the topology of complete Riemannian manifolds of low dimension with nonnegative scalar or Ricci curvature. In general, it is a problem of basic interest to study the topological and geometrical relationships between a minimal submanifold and the manifold in which it is immersed. A well-known result of Frankel [1] asserts that if $\Sigma$ is a compact immersed minimal hypersurface in a Riemannian manifold $M$ with strictly positive Ricci curvature then the homomorphism of fundamental groups: $\Pi(\Sigma) \rightarrow \Pi(M)$ induced by inclusion is onto. As the product of spheres $S^{1} \times S^{2}$ shows, the conclusion of Frankel's theorem is false if the Ricci curvature is only assumed to be nonnegative. The purpose of this paper is to study the rigidity of Frankel's theorem, i.e. to study the extent to which Frankel's theorem can fail when the Ricci curvature is only assumed to be nonnegative. Our main theorem, stated below, shows that the theorem can fail only under special circumstances.

THEOREM. Let $M$ be a complete $n$-dimensional Riemannian manifold with nonnegative Ricci curvature. Let $\varphi: \Lambda \rightarrow M$ be a minimal immersion, where $\Lambda$ is a compact $(n-1)$-dimensional manifold, and let $\Sigma=\varphi(\Lambda)$. Consider the homomorphism of fundamental groups $i_{*}: \Pi(\Sigma) \rightarrow \Pi(M)$ induced by the inclusion map $i: \Sigma \rightarrow M$. Then either (a) below holds, or $\Sigma$ is an imbedded totally geodesic submanifold of $M$ and one of (b)-(e) holds.

(a) $i_{*}$ is onto.

(b) $\Pi(M) / i_{*}(\Pi(\Sigma))=Z_{2}$. $\Sigma$ separates $M$, and the closure of one of the components of $M-\Sigma$ has a double covering which is isometric to $[0, L] \times \Sigma$. 
(c) $M$ is isometric to the (possibly twisted) product of $S^{1}$ and $\Sigma$. (More precisely, $\Sigma$ is two-sided and $M-\Sigma$ is isometric to $(0, L) \times \Sigma$, where the isometry is given by exponentiating normally from one side of $\Sigma$ to the other).

(d) $\Sigma$ separates $M$, and a double covering of $M$ is isometric to the ( possibly twisted) product of $S^{1}$ and $\Sigma$.

(e) $\Sigma$ is one-sided, and a double covering of $M$ is isometric to the ( possibly twisted) product of $S^{1}$ and a double covering of $\Sigma$.

In addition, if $i_{*}$ is not onto, the Ricci curvature vanishes on all vectors orthogonal to $\Sigma$.

We remark that if one assumes $M$ and $\Sigma$ are orientable then case (e), and only case (e), can be eliminated. There are simple models illustrating each of the possibilities (a)-(e).

Case (a). $M=$ projective 2-space realized as $S^{2}$ with antipodal points identified; $\Sigma=$ equator with antipodal points identified.

Case (b). $M=$ projective 2 -space realized as $S^{2}$ flattened near the equator, with antipodal points identified; $\Sigma=$ circle of latitude near the equator.

Case (c) (a twisted example). $M=$ flat Klein bottle realized as a square with horizontal sides identified in the opposite direction, and vertical sides identified in the same direction; $\Sigma=S^{1}$ realized as a horizontal line segment joining the vertical edges.

Case (d). $M$ as in case (c); $\Sigma=S^{1}$ realized as two vertical line segments symmetrically spaced around the central vertical line.

Case (e). $M$ as in case (c); $\Sigma=S^{1}$ realized as the central vertical line.

Lawson ([4], Theorem 1) has obtained a result closely related to Frankel's. He proves that if $M$ is a compact connected Riemannian manifold with mean convex boundary $\partial M$ and if $M$ has positive Ricci curvature then $\partial M$ is connected and the homomorphism $\Pi(\partial M) \rightarrow \Pi(M)$ induced by inclusion is onto. Meeks ([6], Proposition 1) has considered the rigidity of Lawson's result in the case $M$ is flat. He shows $i_{*}: \Pi(\partial M) \rightarrow$ $\Pi(M)$ is onto unless $\partial M$ is totally geodesic, and observes that if $i_{*}$ is not onto then $\Pi(M) / i_{*}(\Pi(\partial M))=Z_{2}$. The proof of our main theorem makes use of an appropriately generalized version of Meek's result. We 
would also like to mention here the paper of Meyer [8] which is related to various aspects of our work.

2. The proof. We begin with a number of preliminary results. The first result is the principal geometric tool upon which the proof of the main theorem relies.

LEMMA 1. Let $M$ be a complete connected Riemannian manifold with mean convex boundary $\partial M$ which has at least two components, $\Sigma_{1}$ and $\Sigma_{2}$. If $M$ has nonnegative Ricci curvature and $\Sigma_{1}$ is compact then $\partial M=\Sigma_{1} \cup \Sigma_{2}$ and $M$ is isometric to $[0, L] \times \Sigma_{1}$, where the isometry is given by exponentiating normally from $\Sigma_{1}$ to $\Sigma_{2}$. In particular, $\Sigma_{1}$ and $\Sigma_{2}$ are isometric.

Ichida [2] and Kasue [3] have given proofs of this result by different methods. The proof in a less general setting is implicitly discussed in Meeks ([6], Proposition 1). The following lemma generalizes Proposition 1 in Meeks [6] and Theorem 1 in Lawson [4]. The idea of the proof is similar to that of Proposition 1 in Meeks [6].

LEMMA 2. Let $M$ be a complete connected Riemannian manifold with compact connected boundary $\Sigma$. Assume $M$ has nonnegative Ricci curvature and $\Sigma$ is mean convex. Then, either

(a) $i_{*}: \Pi(\Sigma) \rightarrow \Pi(M)$ is onto, or

(b) $\Pi(M) / i_{*}(\Pi(\Sigma))=Z_{2}$, and $M$ has a double covering which is isometric to $[0, L] \times \Sigma$. In particular, $\Sigma$ is totally geodesic and the Ricci curvature vanishes on all vectors orthogonal to $\Sigma$.

Proof of Lemma 2. The proof is an application of Lemma 1. Let $(\tilde{M}, p)$ be the Riemannian covering manifold of $M$ such that $p_{*}(\Pi(\tilde{M}, \tilde{s}))=i_{*}(\Pi(\Sigma, s))$. Let $\tilde{\Sigma}_{0}$ be the component of $p^{-1}(\Sigma)$ passing through $\tilde{s}$. We mention two simple properties of $(\tilde{M}, p)$ : (i) Any loop in $\tilde{M}$ based at $\tilde{s}$ is fixed end point homotopic to a loop in $\tilde{\Sigma}_{0}$ based at $\tilde{s}$, and (ii) $\left.p\right|_{\tilde{\Sigma}_{0}}: \tilde{\Sigma}_{0} \rightarrow \Sigma$ is injective (and hence an isometry).

Suppose $i_{*}$ is not onto. Then there exists a component $\tilde{\Sigma}$ of $\partial \tilde{M}$ distinct from $\tilde{\Sigma}_{0}$. By Lemma $1, \partial \tilde{M}=\tilde{\Sigma}_{0} \cup \tilde{\Sigma}$, and $\tilde{M}$ is isometric to $[0, L] \times \tilde{\Sigma}_{0}$. It follows that $\Sigma$ is totally geodesic and that the Ricci curvature vanishes on vectors normal to $\Sigma$. Since $\left.p\right|_{\tilde{\Sigma}}: \tilde{\Sigma} \rightarrow \Sigma$ is a covering map, we have $\operatorname{vol}(\tilde{\Sigma})=k \operatorname{vol}(\Sigma)$, where $k$ is the number of sheets of the covering $\left(\tilde{\Sigma},\left.p\right|_{\tilde{\Sigma}}\right)$. Thus, since $\tilde{\Sigma}$ is isometric to $\tilde{\Sigma}_{0}$, and $\tilde{\Sigma}_{0}$ is isometric to $\Sigma,\left(\tilde{\Sigma},\left.p\right|_{\tilde{\Sigma}}\right)$ must be a single sheeted covering of $\Sigma$, i.e. $p \mid \tilde{\Sigma}: \tilde{\Sigma} \rightarrow \Sigma$ must be injective. 
Since $p^{-1}(\Sigma)$ consists of two copies of $\Sigma$ we have that $(\tilde{M}, p)$ is a double covering of $M$. Statement (b) now follows from the fact that, for regular coverings, the group of deck transformations (which in the present case is $\left.Z_{2}\right)$ is isomorphic to $\Pi(M, s) / p_{*} \Pi(\tilde{M}, \tilde{s})$.

The following lemma is the basic imbeddedness result needed for the proof of the main theorem.

LEMMA 3. Let $M$ be an n-dimensional complete connected Riemannian manifold with nonnegative Ricci curvature. Let $\varphi_{\alpha}: \Lambda_{\alpha} \rightarrow M, \alpha=1,2$, be minimal immersions such that $\operatorname{dim} \Lambda_{\alpha}=n-1$, and let $\Sigma_{\alpha}=\varphi_{\alpha}\left(\Lambda_{\alpha}\right)$. Assume $\Lambda_{1}$ is compact and $\Sigma_{2}$ is closed. If $\Sigma_{1}$ and $\Sigma_{2}$ don't meet then they must be totally geodesic imbedded submanifolds of $M$, and $\Sigma_{2}$ is compact.

Proof of Lemma 3. The proof is an application of the maximum principle for the mean curvature equation (cf. Ichida [2]). Without loss of generality we can assume that $\varphi_{1}: \Lambda_{1} \rightarrow M$ is a two-sided immersion, by which we mean there exists a smooth nonvanishing normal vector field defined along $\varphi_{1}$, i.e., there exists a smooth map $N: \Lambda_{1} \rightarrow T M$ such that for each $p \in \Lambda_{1}, N(p)$ is a vector at $T_{\varphi_{1}(p)} M$ orthogonal to $d \varphi_{1}\left(T_{p} \Lambda_{1}\right)$. Indeed, if $\varphi_{1}$ is not two-sided then by standard covering space arguments there exists a two-sheeted covering $\left(\tilde{\Lambda}_{1}, p\right)$ of $\Lambda_{1}$ such that $\varphi_{1} \circ p$ : $\tilde{\Lambda}_{1} \rightarrow M$ is two-sided.

Let $L>0$ be the distance between $\Sigma_{1}$ and $\Sigma_{2}$. Let $\gamma:[0, L] \rightarrow M$ be any unit speed geodesic segment, with $\gamma(0)=q_{1} \in \Sigma_{1}$ and $\gamma(L)=q_{2} \in$ $\Sigma_{2}$, which achieves this minimum. (Since $\Sigma_{1}$ is compact and $\Sigma_{2}$ is closed, at least one such $\gamma$ exists). $\gamma$ strikes $\Sigma_{1}$ and $\Sigma_{2}$ orthogonally. In particular, there can be no transverse self-intersections at $q_{1}$ and $q_{2}$. For each $\alpha=1,2$, choose a point $p_{\alpha} \in \varphi_{\alpha}^{-1}\left(q_{\alpha}\right)$ and a neighborhood $U_{\alpha}$ of $p_{\alpha}$ such that $\left.\varphi_{\alpha}\right|_{U_{\alpha}}: U_{\alpha} \rightarrow M$ is an imbedding., Set $V_{\alpha}=\varphi_{\alpha}\left(U_{\alpha}\right)$. By the lemma in Section 3 of Ichida [2], whose proof is an application of the maximum principle for the mean curvature equation, there is a neighborhood $W_{1} \subset$ $U_{1}$ of $p_{1}$ such that $\varphi_{1}\left(W_{1}\right)$ is totally geodesic and the map $E: W_{1} \rightarrow M$ defined by $E(p)=\exp _{\varphi_{1}(p)} L N(p)$, where $N$ is the normal vector field defined along $\varphi_{1}$ which equals $\gamma^{\prime}(0)$ at $q_{1}$, is a smooth isometric imbedding such that $E\left(W_{1}\right) \subset V_{2}$ and $E\left(W_{1}\right)$ is totally geodesic. (The metric on $W_{1}$ is the pullback of the induced metric on $\left.V_{1}\right)$.

Now fix a geodesic segment $\gamma:[0, L] \rightarrow M$ which minimizes the distance from $\Sigma_{1}$ to $\Sigma_{2}$, and let $N$ be the normal vector field along $\varphi_{1}$ which agrees with $\gamma^{\prime}(0)$ at $\gamma(0)$. The set $W=\left\{p \in \Sigma\right.$ : $\exp _{\phi_{1}(p)} L N(p) \in$ $\left.\Sigma_{2}\right\}$ is closed by the continuity of the exponential map, and open by the 
discussion in the previous paragraph. Thus, by connectedness, $W=\Lambda_{1}$ which implies that each geodesic issuing from $\Sigma_{1}$ with initial direction $N$ achieves the minimum distance between $\Sigma_{1}$ and $\Sigma_{2}$. From the discussion in the preceding paragraph $\Sigma_{1}$ is totally geodesic. Furthermore, by another connectedness argument we see that $\Sigma_{2}=\left\{\exp _{\varphi_{1}(p)} L N(p): p \in \Lambda_{1}\right\}$, and hence $\Sigma_{2}$ is compact and totally geodesic, as well.

We have shown that every point in $\Sigma_{1}$ is the minimum distance $L$ from $\Sigma_{2}$ (and vice-versa). Thus, any self-intersections in $\Sigma_{1}$ must be tangential. Let $x_{1}, x_{2} \in \Sigma_{1}$ be such that $\varphi_{1}\left(x_{1}\right)=\varphi_{1}\left(x_{2}\right)=y$. Let $S_{1}, S_{2}$ be neighborhoods of $x_{1}$ and $x_{2}$, respectively, such that $\varphi_{1}: S_{\alpha} \rightarrow M$, $\alpha=1,2$, are imbeddings. Since $\varphi_{1}\left(S_{1}\right)$ and $\varphi_{1}\left(S_{2}\right)$ meet tangentially at $y$ and are totally geodesic, there exist neighborhoods $Q_{\alpha} \subset S_{\alpha}$, of $x_{\alpha}$, $\alpha=1,2$, such that $\varphi_{1}\left(Q_{1}\right)=\varphi_{1}\left(Q_{2}\right)$. Using this observation and the compactness of $\Lambda_{1}$, one can show that for each $p \in \Sigma_{1}$, there exists an open set $o$ in $M$ containing $p$ and an open set $W$ in $\Sigma$ such that $\varphi_{1 \mid W}$ : $W \rightarrow M$ is an imbedding and $\varphi_{1}(W)=\Sigma_{1} \cap o$. It follows that $\Sigma_{1}$ is an imbedded submanifold. A similar argument shows that $\Sigma_{2}$ is imbedded.

COROLlaRY. Let the setting be as in the main theorem. If $i_{*}$ is not onto then $\Sigma$ is an imbedded totally geodesic submanifold of $M$.

Proof of the Corollary. Let $(\tilde{M}, p)$ be the Riemannian covering manifold of $M$ satisfying $p_{*}(\Pi(\tilde{M}, \tilde{s}))=i_{*}(\Pi(\Sigma, s))$. (This covering was introduced in the proof of Lemma 2 , but in a slightly different context. The properties mentioned there hold in the present context as well.) Let $\tilde{\Sigma}_{0}$ be the component of $p^{-1}(\Sigma)$ containing $\tilde{s}$. Since $i_{*}$ is not onto, there exists at least one other component, $\tilde{\Sigma}$, say, of $p^{-1}(\Sigma)$. Using basic covering space theory (see e.g. Massey [5]) there exist immersions $\varphi_{1}$ : $\Lambda \rightarrow \tilde{M}$ and $\varphi_{2}: \tilde{\Lambda} \rightarrow \tilde{M}$, where $\tilde{\Lambda}$ is a manifold covering $\Lambda$, such that $\varphi_{1}(\Lambda)=\tilde{\Sigma}_{0}, \varphi_{2}$ is proper, and $\varphi_{2}(\tilde{\Lambda}) \subset \tilde{\Sigma}$. (Take $\left(\tilde{\Lambda}, p_{1}\right)$ to be the covering manifold of $\Lambda$ satisfying $\left(p_{1}\right)_{*}(\Pi(\tilde{\Lambda}))=\varphi^{-1} *\left(p_{*}(\Pi(\tilde{M}))\right.$.) Hence, by Lemma $3, \tilde{\Sigma}_{0}$ is imbedded and totally geodesic. Since $p$ : $\tilde{M} \rightarrow M$ is a local isometry the same conclusions apply to $\Sigma$.

Proof of the Theorem. In view of the corollary to Lemma 3 it is sufficient to prove the main theorem under the assumption that $\Sigma$ is a compact imbedded minimal hypersurface in $M$.

Assume for the time being that $\Sigma$ is two-sided. Suppose $\Sigma$ does not separate $M$. Since $\Sigma$ is two-sided we can make a "cut" along $\Sigma$ to obtain a manifold with boundary $M^{\prime}$, whose boundary consists of two disjoint copies of $\Sigma, \Sigma_{1}$ and $\Sigma_{2}$ say, such that any curve in $M^{\prime}$ from $\Sigma_{1}$ to $\Sigma_{2}$ 
corresponds to a curve in $M$ from one side of $\Sigma$ to the other. Lemma 1 implies that the normal exponential map $\Phi:[0, L] \times \Sigma_{1} \rightarrow M^{\prime}$, where $L=d\left(\Sigma_{1}, \Sigma_{2}\right)$, is an isometry. Thus, part (c) of the theorem holds.

Suppose now that $\Sigma$ separates $M$. Let $M_{1}$ and $M_{2}$ denote the closures of the two components of $M-\Sigma$. Hence, $M_{1}$ and $M_{2}$ are manifolds with common boundary $\Sigma$. Consider the following diagram of inclusion maps,

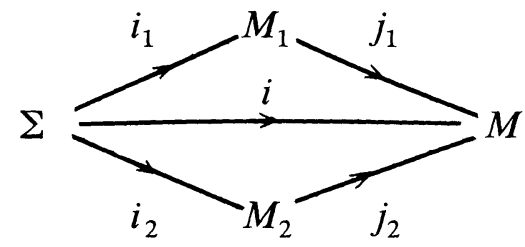

Set $G_{0}=i_{*}(\Pi(\Sigma)), H_{\alpha}=\left(i_{\alpha}\right)_{*}(\Pi(\Sigma)), G_{\alpha}=\left(j_{\alpha}\right)_{*}\left(\Pi\left(M_{\alpha}\right)\right), \alpha=1,2$. By Lemma 2, either $\Pi\left(M_{\alpha}\right)=H_{\alpha}$ or $\Pi\left(M_{\alpha}\right) / H_{\alpha}=Z_{2}$. It then follows easily that either $G_{\alpha}=G_{0}$ or $G_{\alpha} / G_{0}=Z_{2}, \alpha=1,2$.

We now consider several cases. Recall by the Seifert-Van Kampen theorem that $\Pi(M)$ is generated by $G_{1}$ and $G_{2}$.

Case 1. $G_{1}=G_{0}$ and $G_{2}=G_{0}$. In this case, $\Pi(M)=G_{0}$, and statement (a) of the theorem holds.

Case 2. $G_{1} \neq G_{0}$ and $G_{2}=G_{0}$. In this case $\Pi(M)=G_{1}$. Statement (b) of the theorem now follows from the equality, $G_{1} / G_{0}=Z_{2}$, and Lemma 2.

Case 3. $G_{1}=G_{0}$ and $G_{2} \neq G_{0}$. As in Case 2, this leads to statement (b) of the theorem.

Case 4. $G_{1} \neq G_{0}$ and $G_{2} \neq G_{0}$. In this case, $\Pi\left(M_{\alpha}\right) / H_{\alpha}=Z_{2}, \alpha=$ 1,2. For each $\alpha=1,2$, let $\left(\tilde{M}_{\alpha}, p_{\alpha}\right)$ be the covering manifold of $M_{\alpha}$ which satisfies, $\left(p_{\alpha}\right)_{*}\left(\Pi\left(\tilde{M}_{\alpha}\right)\right)=H_{\alpha}$. Then $\left(\tilde{M}_{\alpha}, p_{\alpha}\right)$ is a double covering of $M_{\alpha}$, and $\tilde{M}_{\alpha}$ is a manifold with boundary $p_{\alpha}^{-1}(\Sigma)$ which consists of two copies of $\Sigma$. By appropriately "gluing" together the boundaries of $\tilde{M}_{1}$ and $\tilde{M}_{2}$, we obtain a double covering of $M$ which contains two copies of $\Sigma$, neither of which separates. But this situation was considered at the beginning of the proof. From what was done there, one sees that we are now led to part (d) of the theorem.

Thus if $\Sigma$ is two-sided one of the cases (a)-(d) must hold.

Suppose now that $\Sigma$ is not two-sided. It is then standard, using Mod 2 intersection theory, to construct a two-sheeted covering $(\hat{M}, p)$ of $M$ such that $\left(\hat{\Sigma}=p^{-1}(\Sigma), p_{\mid \hat{\Sigma}}\right)$ is a connected double covering of $\Sigma$ and $\hat{\Sigma}$ is two-sided. In fact this construction actually implies that $\hat{\Sigma}$ separates $\hat{M}$. 
Thus, cases (a)-(d) of the main theorem (and the arguments leading to these cases) apply to $\hat{\Sigma} \subset \hat{M}$. We now observe that if $i_{*}: \Pi(\hat{\Sigma}, \hat{s}) \rightarrow$ $\Pi(\hat{M}, \hat{s})$ is onto, so is $i_{*}: \Pi(\Sigma, s) \rightarrow \Pi(M, s)$ (where $\hat{s} \in p^{-1}(s)$ ). Let $g=\langle\gamma\rangle$ be any element of $\Pi(M, s)$, where $\gamma$ is a loop based at $s$. Let $\hat{\gamma}$ be the lift of $\gamma$ from $\hat{s} \in \hat{\Sigma}$ to $\hat{x} \in \hat{\Sigma}$. Let $\hat{\sigma}$ be a curve in $\hat{\Sigma}$ from $\hat{x}$ to $\hat{s}$. By assumption, $\langle\hat{\gamma} \hat{\sigma}\rangle \in i_{*} \Pi(\hat{\Sigma}, \hat{s})$. Projecting via the covering map gives $\langle\gamma\rangle\langle\sigma\rangle=\langle\gamma \sigma\rangle \in i_{*} \Pi(\Sigma, s)$, where $\sigma=p \circ \hat{\sigma}$. Hence, since $\langle\sigma\rangle \in$ $i_{*} \Pi(\Sigma, s), g=\langle\gamma\rangle \in i_{*} \Pi(\Sigma, s)$. Thus, if case (a) holds for $\hat{\Sigma} \subset \hat{M}$ then case (a) holds for $\Sigma \subset M$. Since the two components of $\hat{M}-\hat{\Sigma}$ are isometric (via a deck transformation), the discussion leading to case (b) in the two-sided setting shows that case (b) is not applicable to $\hat{\Sigma} \subset \hat{M}$. Case (c) is also ruled out because $\hat{\Sigma}$ separates. It remains to consider case (d).

By our earlier arguments which led to case (d) we may assume that there exists a double covering $(\tilde{M}, \varphi)$ of $\hat{M}$ such that $(1) \varphi^{-1}(\hat{\Sigma})$ consists of two disjoint copies, $\tilde{\Sigma}_{1}$ and $\tilde{\Sigma}_{2}$, say, of $\tilde{\Sigma}$ and (2) each component of $\hat{M}-\hat{\Sigma}$ is double covered by a component of $\tilde{M}-\left(\tilde{\Sigma}_{1} \cup \tilde{\Sigma}_{2}\right) .(\tilde{M}, \psi)$, where $\psi=p \circ \varphi$, is a four-sheeted covering of $M$. We use the "cut and paste" method on $\tilde{M}$ to obtain a new double covering of $M$.

Let $\hat{U}$ be a component of $\hat{M}-\hat{\Sigma}$, and let $\tilde{U}$ be the component of $\tilde{M}-\left(\tilde{\Sigma}_{1} \cup \tilde{\Sigma}_{2}\right)$ such that $\left(\tilde{U},\left.\varphi\right|_{\tilde{U}}\right)$ is a double covering of $\hat{U}$. Since $\hat{U}$ is one of the two components of $p^{-1}(M-\Sigma),\left(\hat{U},\left.p\right|_{\hat{U}}\right)$ is a single covering of $M-\Sigma$. Consequently, $\left(\tilde{U},\left.\psi\right|_{\tilde{U}}\right)$ is a double covering of $M-\Sigma$. The closure of $\tilde{U}$, call it $D$, is a manifold with boundary $\tilde{\Sigma}_{1} \cup \tilde{\Sigma}_{2}=\psi^{-1}(\Sigma)$. Let $V$ denote the manifold without boundary obtained from $D$ by identifying the points of $\tilde{\Sigma}_{1}$ and $\tilde{\Sigma}_{2}$ as follows: For all $x_{1} \in \tilde{\Sigma}_{1}, x_{2} \in \tilde{\Sigma}_{2}$, identify $x_{1}$ and $x_{2}$ if and only if $\psi\left(x_{1}\right)=\psi\left(x_{2}\right)$ and $\varphi\left(x_{1}\right) \neq \varphi\left(x_{2}\right)$. Then $V$ (with covering map essentially given by $\psi$ ) is a double covering of $M$ such that the inverse image of $\Sigma$ with respect to the covering map is a double covering of $\Sigma$ which is two-sided, but which does not separate $V$. (To visualize this construction, it is helpful to carry it out explicitly on the example of case (e) given after the statement of the main theorem.) The argument of case (c) applied to the present situation now leads to case (e) of the theorem.

Thus, we have shown that one of the cases (a)-(e) must hold. If $i_{*}$ is not onto then, by the corollary to Lemma $3, \Sigma$ is totally geodesic. The last sentence in the statement of the theorem is a consequence of the product structure in cases (b)-(e).

The following corollary singles out the situation in which $M$ is noncompact. The imbeddedness result needed for this corollary has already been established in Kasue ([3], Theorem 1). 
COROLlaRY. Let $M$ be a complete noncompact n-dimensional Riemannian manifold with nonnegative Ricci curvature. Let $\varphi: \Lambda \rightarrow M$ be a minimal immersion, where $\Lambda$ is a compact $(n-1)$-dimensional manifold. Then $\Sigma=\varphi(\Lambda)$ is an imbedded totally geodesic submanifold of $M$ and one of the following must hold.

(1) $M$ is isometric to $\mathbf{R} \times \Sigma$.

(2) $M$ has one end, and there is a double covering of $M$ which is isometric to the product of $\mathbf{R}$ and a double covering of $\Sigma$.

(3) $M$ has one end and $\Sigma$ separates $M$. The closure of the component of $M-\Sigma$ containing this end is isometric to $[0, \infty) \times \Sigma$ and,

$$
\Pi(M) / i_{*}(\Pi(\Sigma))=1 \text { or } Z_{2} \text {. }
$$

Proof. We begin by observing that $(*)$ holds in all cases. Indeed, since $M$ is noncompact only cases (a) and (b) of the main theorem are applicable. (However note in cases (1) and (2), $i_{*}$ is actually onto.) By Theorem 1 in Kasue [3], $\Sigma$ is imbedded and totally geodesic. Suppose $\Sigma$ is two-sided. Then $\Sigma$ must separate $M$ (otherwise $M$ would be compact as in case (c) of the main theorem). $M$ has at most two ends. If $M$ has two ends then (as observed in Meeks, Simon and Yau [7], §9) the splitting theorem of Cheeger and Gromoll implies that (1) holds. If $M$ has only one end then Theorem $\mathrm{C}$ in Kasue [3] implies that the closure of the component containing this end is isometric to $[0, \infty) \times \Sigma$.

Suppose now that $\Sigma$ is not two-sided. As discussed earlier, there is a double covering $(\hat{M}, p)$ of $M$ such that $\hat{\Sigma}=p^{-1}(\Sigma)$ is connected and separates $\hat{M}$. Furthermore each component of $\hat{M}-\hat{\Sigma}$ must be noncompact and hence $\hat{M}$ has two ends. Case (1) then applies to $\hat{\Sigma} \subset \hat{M}$, and thus case (2) holds.

Again, there are simple models illustrating each of the three cases. For instance, the nontrivial line bundle over $S^{1}$ (i.e. the Möbius band) is an example illustrating case (2).

As a final remark note that if $M$ is three dimensional and satisfies the hypotheses of the corollary then, by Gauss-Bonnet, $\Sigma$ must have genus zero or one. All three cases can occur in dimension three. (In the case $M$ is orientable, Theorem 6 in [7] overlooks the possibility that case (3) can occur.)

The author gratefully acknowledges helpful conversations with Ted Frankel, Joel Hass, Marvin Mielke, and Lucio Rodriguez. 


\section{REFERENCES}

[1] T. Frankel, On the fundamental group of a compact minimal submanifold, Ann. Math., 83 (1966), 68-73.

[2] R. Ichida, Riemannian Manifolds with compact boundary, Yokohama Math. J., 29 (1981).

[3] A. Kasue, Ricci curvature, geodesics and some geometric properties of Riemannian manifolds with boundary, J. Math. Soc. Japan, 35 (1983), 117-131.

[4] H. B. Lawson, The unknottedness of minimal embeddings, Invent. Math., 11 (1970), 183-187.

[5] William Massey, Algebraic Topology: An Introduction, Springer-Verlag, New York, 1977.

[6] W. H. Meeks, The topological uniqueness of minimal surfaces in Euclidean space, Topology, 20 (1981), 389-410.

[7] W. H. Meeks, L. Simon, and S. T. Yau, Embedded minimal surfaces, exotic spheres, and manifolds with positive Ricci curvature, Ann. Math., 116 (1982), 621-659.

[8] D. Meyer, Sur les hypersurfaces minimales des varietes Riemanniennes a coubure de Ricci positive ou nulle, Bull. Soc. Math. France, 111 (1983), 359-366.

Received June 3, 1985 and in revised form December 26, 1985.

UNIVERSITY OF MIAMI

Coral GaBles, FL 33124 



\title{
PACIFIC JOURNAL OF MATHEMATICS \\ EDITORS
}

\section{S. VARADARAJAN}

(Managing Editor)

University of California

Los Angeles, CA 90024

HERBERT CLEMENS

University of Utah

Salt Lake City, UT 84112

R. FINN

Stanford University

Stanford, CA 94305
HERMANN FLASCHKA

University of Arizona

Tucson, AZ 85721

RAMESH A. GANGOLLI

University of Washington Seattle, WA 98195

VAughan F. R. Jones

University of California

Berkeley, CA 94720

ROBION KIRBY

University of California

Berkeley, CA 94720
C. C. MOORE

University of California

Berkeley, CA 94720

H. SAMELSON

Stanford University

Stanford, CA 94305

HAROLD STARK

University of California, San Diego

La Jolla, CA 92093

\section{ASSOCIATE EDITORS}

\author{
R. ARENS \\ E. F. BECKENBACH \\ B. H. NEUMANN \\ F. WOLF \\ K. YoshIDA \\ (1906-1982)

\section{SUPPORTING INS'TITUTIONS} \\ UNIVERSITY OF ARIZONA \\ UNIVERSITY OF BRITISH COLUMBIA \\ CALIFORNIA INSTITUTE OF TECHNOLOGY \\ UNIVERSITY OF CALIFORNIA \\ MONTANA STATE UNIVERSITY \\ UNIVERSITY OF NEVADA, RENO \\ NEW MEXICO STATE UNIVERSITY \\ OREGON STATE UNIVERSITY \\ UNIVERSITY OF OREGON \\ UNIVERSITY OF SOUTHERN CALIFORNIA \\ STANFORD UNIVERSITY \\ UNIVERSITY OF HAWAII \\ UNIVERSITY OF TOKYO \\ UNIVERSITY OF UTAH \\ WASHINGTON STATE UNIVERSITY \\ UNIVERSITY OF WASHINGTON
}

The Supporting Institutions listed above contribute to the cost of publication of this Journal, but they are not owners or publishers and have no responsibility for its content or policies.

Mathematical papers intended for publication in the Pacific Journal of Mathematics should be in typed form or offset-reproduced (not dittoed), double spaced with large margins. Please do not use built up fractions in the text of the manuscript. However, you may use them in the displayed equations. Underline Greek letters in red, German in green, and script in blue. The first paragraph must be capable of being used separately as a synopsis of the entire paper. In particular it should contain no bibliographic references. Please propose a heading for the odd numbered pages of less than 35 characters. Manuscripts, in triplicate, may be sent to any one of the editors. Please classify according to the scheme of Math. Reviews, Index to Vol. 39. Supply name and address of author to whom proofs should be sent. All other communications should be addressed to the managing editor, or Elaine Barth, University of California, Los Angeles, California 90024.

There are page-charges associated with articles appearing in the Pacific Journal of Mathematics. These charges are expected to be paid by the author's University, Government Agency or Company. If the author or authors do not have access to such Institutional support these charges are waived. Single authors will receive 50 free reprints; joint authors will receive a total of 100 free reprints. Additional copies may be obtained at cost in multiples of 50 .

The Pacific Journal of Mathematics is issued monthly as of January 1966. Regular subscription rate: $\$ 190.00$ a year (5 Vols., 10 issues). Special rate: $\$ 95.00$ a year to individual members of supporting institutions.

Subscriptions, orders for numbers issued in the last three calendar years, and changes of address should be sent to Pacific Journal of Mathematics, P.O. Box 969, Carmel Valley, CA 93924, U.S.A. Old back numbers obtainable from Kraus Periodicals Co., Route 100, Millwood, NY 10546.

The Pacific Journal of Mathematics at P.O. Box 969, Carmel Valley, CA 93924 (ISSN 0030-8730) publishes 5 volumes per year. Application to mail at Second-class postage rates is pending at Carmel Valley, California, and additional mailing offices. Postmaster: send address changes to Pacific Journal of Mathematics, P.O. Box 969, Carmel Valley, CA 93924.

PUBLISHED BY PACIFIC JOURNAL OF MATHEMATICS, A NON-PROFIT CORPORATION Copyright (c) 1987 by Pacific Journal of Mathematics 


\section{Pacific Journal of Mathematics}

\section{Vol. 126, No. 2 December, 1987}

Charles James Keith Batty, Derivations on the line and flows along orbits

Paul Erdốs, Adolf J. Hildebrand, Andrew Odlyzko, Paul Pudaite and

Bruce Reznick, The asymptotic behavior of a family of sequences . . . 227

Gregory James Galloway, A note on the fundamental group of a compact

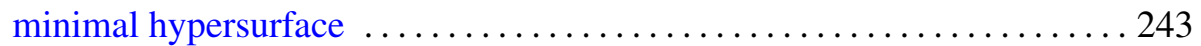

Nigel Higson, A characterization of $K K$-theory $\ldots \ldots \ldots \ldots \ldots \ldots \ldots \ldots 253$

Anthony To-Ming Lau and Wataru Takahashi, Weak convergence and nonlinear ergodic theorems for reversible semigroups of nonexpansive mappings

Pere Menal and Juame Moncasi, Lifting units in self-injective rings and an

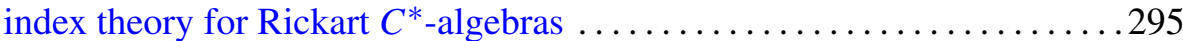

Yoshimi Saito, Schrödinger operators with a nonspherical radiation condition

Larry Smith, Realizing certain polynomial algebras as cohomology rings of

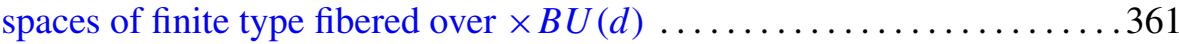

Carl E. Swenson and Calvin T. Long, Necessary and sufficient conditions for simple $A$-bases . . . . . . . . . . . . . . . . . . . . . . . . . . . . . . . 379

Kenneth Giovanni Valente, The $p$-primes of a commutative ring 385 